\title{
Prevalence of autoantibodies against Ras-like GTPases, RalA, in patients with gastric cancer
}

\author{
TATSUKI NANAMI $^{1}$, ISAMU HOSHINO ${ }^{2}$, MASAAKI ITO ${ }^{1}$, \\ SATOSHI YAJIMA ${ }^{1}$, YOKO OSHIMA ${ }^{1}$, TAKASHI SUZUKI $^{1}$, FUMIAKI SHIRATORI $^{1}$, \\ YOSHIHIRO NABEYA ${ }^{2}$, KIMIHIKO FUNAHASHI ${ }^{1}$ and HIDEAKI SHIMADA ${ }^{1,3}$ \\ ${ }^{1}$ Department of Surgery, School of Medicine, Toho University, Tokyo $143-8541 ;{ }^{2}$ Division of Gastroenterological Surgery, \\ Chiba Cancer Center, Chiba 260-8717; ${ }^{3}$ Department of Gastroenterological Surgery and Clinical Oncology, \\ Graduate School of Medicine, Toho University, Tokyo 143-8541, Japan
}

Received December 12, 2019; Accepted June 3, 2020

DOI: $10.3892 / \mathrm{mco} .2020 .2098$

\begin{abstract}
Ras-like GTPases, RalA and RalB, are members of the Ras superfamily of small GTPases. RalA expression has been shown to be associated with aggressive clinicopathological characteristics and progression in cancer. RalA protein has been shown to be involved in immune reactions in some patients with cancer; however, the clinicopathological significance of serum RalA antibody in patients with gastric cancer has not been investigated. Serum samples of 291 patients with gastric cancer and 73 healthy controls were analyzed for serum RalA antibody using enzyme-linked immunosorbent assay. A cut-off optical density value was fixed at 0.255 (mean of control +2 standard deviations). The clinicopathological and prognostic significance of s-RalA-Abs was evaluated. The positivity rate for serum RalA antibody (s-RalA-Abs) was 15\%. The presence of serum RalA antibody was higher in younger patients compared with elderly patients, however this tendency was not statistically significant. s-RalA-Abs was not associated with tumor stage. Since s-RalA-Abs was independent of CEA (carcinoembryonic antigen) and carbohydrate antigen 19-9 (CA19-9), the combination of s-RalA-Abs with CEA and CA19-9 significantly increased the detection rate of gastric cancer at each tumor stage. Patients who were tested positive for s-RalA-Abs showed poor long-term survival; however, this association was not statistically significant by multivariate analysis. In conclusion, s-RalA-Abs may be a candidate serum marker for gastric cancer, when used
\end{abstract}

Correspondence to: Professor Hideaki Shimada, Department of Gastroenterological Surgery and Clinical Oncology, Graduate School of Medicine, Toho University, 6-11-1 Omori-Nishi, Ota-ku, Tokyo 143-8541, Japan

E-mail: hideaki.shimada@med.toho-u.ac.jp

Abbreviations: CEA, carcinoembryonic antigen; CA19-9, carbohydrate antigen 19-9; s-RalA-Abs, serum RalA antibody; PBS, phosphate-buffered saline; TNM, Tumor-Node-Metastasis classification

Key words: RalA, gastric cancer, autoantibody, tumor marker in combination with CEA and/or CA19-9. Additionally, the presence of s-RalA-Abs, in combination with CEA and/or CA19-9, was associated with poor survival in patients with gastric cancer.

\section{Introduction}

Ras/phosphoinositide 3-kinase (PI3K)/AKT-associated factors were one of the key molecules involved in the epithelial-to-mesenchymal transition of gastric cancer cells (1). Ras-like GTPases, RalA and RalB, are members of the Ras superfamily of small GTPases. These GTPases are aberrantly induced during tumorigenesis by oncogenic Ras (2). RalA and RalB have been reported as key cancer phenotypic markers and biomarkers of cellular migration, invasion and metastasis $(3,4)$. RalA expression has been shown to be associated with aggressive clinicopathological characteristics and progression in squamous cell carcinoma (3,4). In gastric cancer, Ajani et al (5) reported that Gal-3 induced c-MYC expression through increased RalA activity and an enhanced YAP1/RalA/RalBP complex to confer an aggressive phenotype.

Some IgG autoantibodies have been found to respond to tumor-associated antigens in the sera of patients with cancer, even at the early stages $(6,7)$. Since RalA is a tumor antigen, autoantibodies against RalA (s-RalA-Abs) have been reported as potential biomarkers for hepatocellular (8), esophageal (9), colorectal (10), breast (11) and ovarian (12) carcinoma. Although the role of other autoantibodies has been investigated in patients with gastric cancer (13), the significance of the clinicopathological and prognostic impact of s-RalA-Abs has not yet been demonstrated.

Therefore, the clinicopathological significance and prognostic value of preoperative s-RalA-Abs levels were evaluated in patients with gastric cancer who underwent radical surgery.

\section{Patients and methods}

Collection of sera. Pre-treatment serum samples were obtained from 291 patients with histologically proven gastric adenocarcinoma and from 73 healthy individuals. Double cancer was excluded. All patients with gastric cancer were surgically treated (between July 2011 and July 2013) at the Toho University Omori 


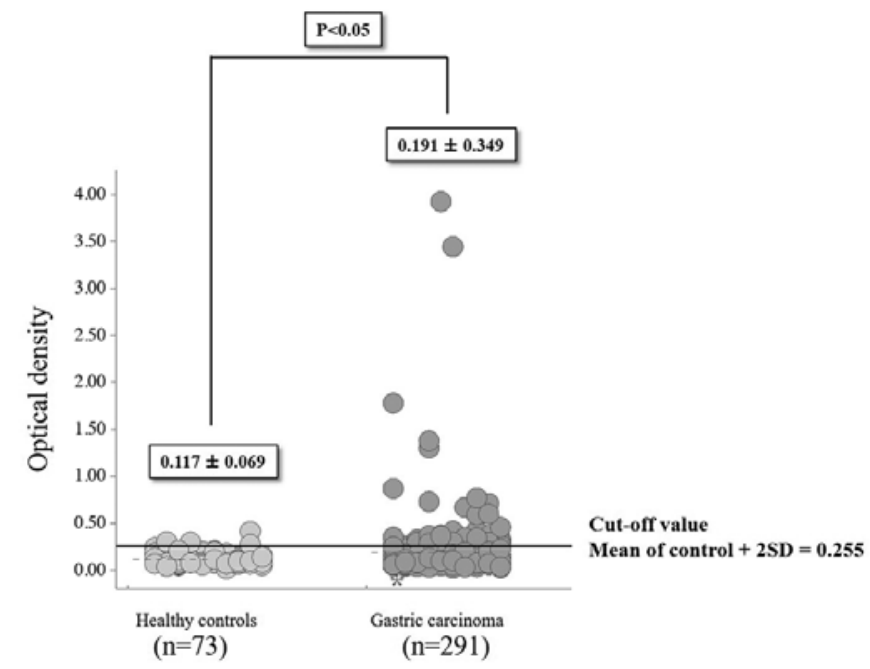

Figure 1. Distribution of serum anti-RalA antibody titers in 291 patients with gastric cancer and 73 healthy controls. The position of the cut-off value (mean of healthy controls $+2 \mathrm{SD}=0.255$ ) is shown. The $\mathrm{P}$ value was calculated by Mann-Whitney U test.

Hospital $(n=76)$ and the Chiba Cancer Center $(n=215)$. Among these, 184 were diagnosed with stage I, 28 with stage II, 29 with stage III, and 50 with stage IV gastric cancer. The patients included 201 men and 90 women (mean age, 67.5 years; range, 36-93 years). Written informed consent was obtained from all patients. The samples were anonymized. Each serum sample was centrifuged at $3,000 \mathrm{x} \mathrm{g}$, at room temperature for $5 \mathrm{~min}$, and the resulting supernatant was stored at $-80^{\circ} \mathrm{C}$ until further analysis. Due care was taken to avoid the repeated thawing and freezing of samples. The present study was approved by the institutional review boards at the Chiba Cancer Center (approval no. \#21-26) and the Toho University School of Medicine (approval nos. \#22-112 and \#22-047).

Purification of recombinant RalA and enzyme-linked immunosorbent assay (ELISA) to detect s-RalA-Abs. RalA construct inserted in pET28 plasmid and expressing the $\mathrm{N}$-terminal His-tagged protein was provided by Dr Jian-Ying Zhang (The University of Texas, El Paso, TX). The details of this procedure have been described previously (9). Sera from patients and healthy controls were analyzed by the previously established ELISA (9). Briefly, purified recombinant proteins were placed in 96-well microtiter plates (Nunc MaxiSorp; Thermo Fisher Scientific, Inc.). RalA was diluted in phosphate-buffered saline (PBS) to a final concentration of $1.0 \mu \mathrm{g} / \mathrm{ml}$ and added to the plates (100 $\mu \mathrm{l} /$ well), which were then incubated overnight at $4^{\circ} \mathrm{C}$. PBS was used as a control. After two washes with PBS, proteins were blocked using $200 \mu \mathrm{l}$ of PBS, containing $1 \%$ bovine serum albumin and $5 \%$ sucrose, at room temperature for $3 \mathrm{~h}$. All human sera were diluted (1:100) in PBS containing $0.15 \%$ Tween-20, $1 \%$ casein, and $0.2 \mathrm{mg} / \mathrm{ml}$ E. coli extract. Then, $100 \mu \mathrm{l}$ diluted sera was added to each RalA- or PBS-coated well and incubated at room temperature, while agitating at $250 \mathrm{rpm}$ for $60 \mathrm{~min}$. After four washes with PBS containing 0.05\% Tween-20 (PBST), $100 \mu \mathrm{l}$ horseradish peroxidase-conjugated antihuman $\operatorname{IgG}(1: 5,000$; Medical \& Biological Laboratories Co., Ltd), diluted in $20 \mathrm{mM}$ 2-[4-(2-hydroxyethyl)-1-piperazinyl] ethanesulfonic acid,
Table I. Comparisons of clinicopathological characteristics and conventional serum markers of patients with serum RalA antibodies.

\begin{tabular}{|c|c|c|c|}
\hline Variables (n) & $\begin{array}{c}\text { s-RalA-Abs- } \\
\text { positive } \\
(\mathrm{n}=43) \%\end{array}$ & $\begin{array}{c}\text { s-RalA-Abs- } \\
\text { negative } \\
(n=248)\end{array}$ & P-values \\
\hline Sex & & & 0.372 \\
\hline Male (201) & $27(13)$ & 174 & \\
\hline Female (90) & $16(18)$ & 74 & \\
\hline Age, years & & & 0.089 \\
\hline$<65(182)$ & $32(18)$ & 150 & \\
\hline$\geqq 65$ (109) & $11(10)$ & 98 & \\
\hline TNM stage & & & 0.359 \\
\hline I+II (212) & $34(16)$ & 178 & \\
\hline III+IV (79) & $9(11)$ & 70 & \\
\hline Depth & & & 0.774 \\
\hline T1+T2 (195) & $28(14)$ & 167 & \\
\hline T3+T4 (96) & $15(16)$ & 81 & \\
\hline Lymph node $^{\mathrm{a}}$ & & & 0.125 \\
\hline Negative (185) & $32(17)$ & 153 & \\
\hline Positive (105) & $11(10)$ & 94 & \\
\hline $\mathrm{CEA}^{\mathrm{a}}$ & & & 1 \\
\hline Negative (226) & $33(15)$ & 193 & \\
\hline Positive (45) & $6(13)$ & 39 & \\
\hline CA19-9a & & & 0.318 \\
\hline Negative (231) & $36(16)$ & 195 & \\
\hline Positive (36) & $3(8)$ & 33 & \\
\hline Tumor size ${ }^{a}$ & & & 0.327 \\
\hline$<5 \mathrm{~cm}(126)$ & $16(12)$ & 110 & \\
\hline$\geqq 5 \mathrm{~cm}(67)$ & $12(17)$ & 55 & \\
\hline $\mathrm{Ly}^{\mathrm{a}}$ & & & 0.520 \\
\hline ly (-) (102) & $16(15)$ & 86 & \\
\hline ly $(+)(96)$ & $12(12)$ & 84 & \\
\hline$v^{a}$ & & & 0.012 \\
\hline v (-) (106) & $21(19)$ & 85 & \\
\hline $\mathrm{v}(+)(93)$ & $7(7)$ & 86 & \\
\hline
\end{tabular}

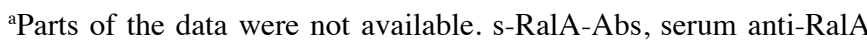
antibodies; TNM, Tumor-Node-Metastasis; CEA, carcinoembryonic antigen; CA19-9; carbohydrate antigen 19-9; Ly, lymphatic invasion; $\mathrm{V}$, venous invasion.

$135 \mathrm{mM} \mathrm{NaCl}, 1 \%$ Bovine serum albumin and $0.1 \%$ hydroxiphenylacetic acid, was added to each well as a secondary antibody. The plates were incubated at room temperature, while agitating at $250 \mathrm{rpm}$ for $60 \mathrm{~min}$. The wells were washed four times with PBST buffer, and autoantibodies were detected by adding $100 \mu 1$ of 3,3',5,5'-tetramethylbenzidine substrate. After incubation at room temperature for $30 \mathrm{~min}$, the reaction was terminated by adding $0.25 \mathrm{~N} \mathrm{H}_{2} \mathrm{SO}_{4}(100 \mu \mathrm{l} /$ well $)$. Absorbance was measured at $450 \mathrm{~nm}$ using the SUNRISE Microplate Reader (Tecan Japan Co., Ltd). RalA signals were determined by calculating the difference between the absorbance values 
for wells containing RalA and PBS. Serum carcinoembryonic antigen (CEA) and carbohydrate antigen 19-9 (CA19-9) markers were also evaluated as described previously (14).

Statistical analyses. Mann-Whitney U test and Fisher's exact probability test, were used to examine the differences between two groups, and Kruskal-Wallis test and Steel-Dwass test were used to compare multiple comparisons. Clinicopathological parameters associated with overall survival were evaluated by univariate analyses using log-rank test based on the Kaplan-Meier survival curves. Multivariate analyses were performed using the Cox proportional hazards model. All statistical analyses were performed using EZR (Saitama Medical Centre, Jichi Medical University; Saitama, Japan) (15), which is a graphical user interface for $\mathrm{R}$ (The $\mathrm{R}$ Foundation for Statistical Computing; version 2.13.0). $\mathrm{P}<0.05$ was considered to indicate a statistically significant difference.

\section{Results}

$s$-RalA-Ab titer. The optical density (mean \pm standard deviation) of s-RalA-Abs was significantly higher $(\mathrm{P}<0.05)$ for the 291 patients with gastric cancer $(0.191 \pm 0.349)$ compared with 73 healthy controls $(0.117 \pm 0.069$; Fig. 1$)$. s-RalA-Ab levels were divided into two groups: Normal optical density values, below the cut-off level of 0.255 (calculated as mean +2 standard deviations of the values in healthy controls) and abnormal or positive values that were 0.255 or higher. The overall positivity rate for s-RalA-Abs was 15\% (43 of 291 patients).

Clinicopathological characteristics, conventional serum markers, and presence of s-RalA-Abs. No statistically significant differences were observed between s-RalA-Abs-positive and s-RalA-Abs-negative patients with respect to sex, age, Tumor-Node-Metastasis classification (TNM) stage (16), tumor depth and lymph node status (Table I). Moreover, no association of s-RalA-Abs with other tumor markers (CEA and CA19-9) was observed (Table I). Although the differences were not statistically significant, lower positivity rates for s-RalA-Abs was shown in T1/T2 tumors and node-positive tumors compared with others. Venous invasion was significantly associated with s-RalA-Abs $(\mathrm{P}<0.05)$. Lymphatic invasion and tumor size were not associated with s-RalA-Abs.

The positivity rates for various markers in patients with gastric cancer were 15\% (43/291) for s-RalA-Abs, $17 \%$ (45/271) for CEA, and 14\% (36/267) for CA19-9 (CEA and CA19-9 were not measured in all cases). A total of 19 patients were positive for both CEA and CA19-9, whereas only six were positive for both s-RalA-Abs and CEA, and three were positive for both s-RalA-Abs and CA19-9 (Fig. 2). Thus, the positivity rates were significantly higher for the combination assay with all the three markers [98 of 291 (34\%)] compared with the two conventional markers-CEA and/or CA19-9 [62 of $291(21 \%)$ ] $(\mathrm{P}<0.01)$. Although the positivity rates for CEA and CA19-9 gradually increased with an increase in the tumor stage, the positivity rates for s-RalA-Abs seemed to be similar at all stages (Fig. 3). In addition, since s-RalA-Abs was independent of CEA and CA19-9, the combination of s-RalA-Abs with CEA and CA19-9 increased the detection rate for gastric cancer at each tumor stage (Fig. 4).

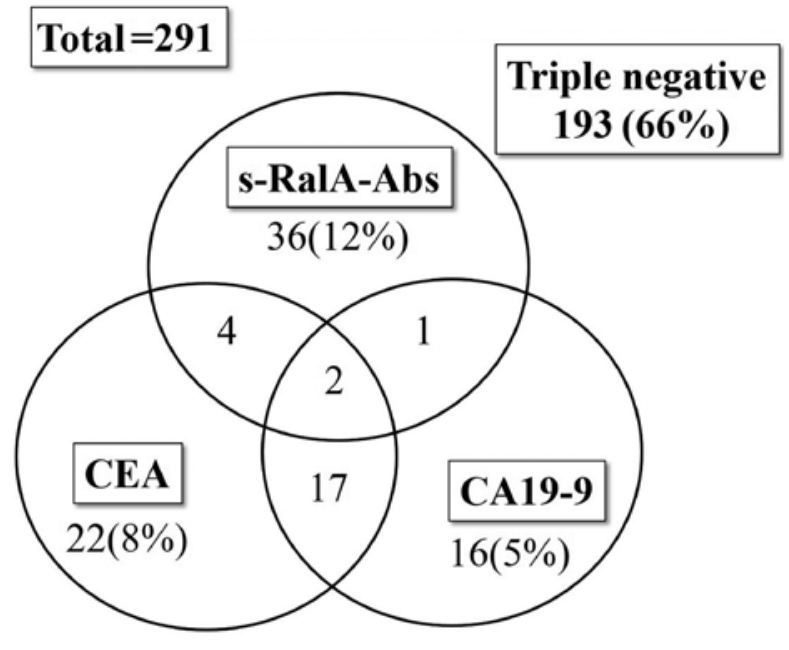

Figure 2. Number of patients positive for each of the tumor markers: s-RalA-Abs, CEA and CA19-9. s-RalA-Abs, serum anti-RalA antibodies; CEA, carcinoembryonic antigen; CA19-9; carbohydrate antigen 19-9.

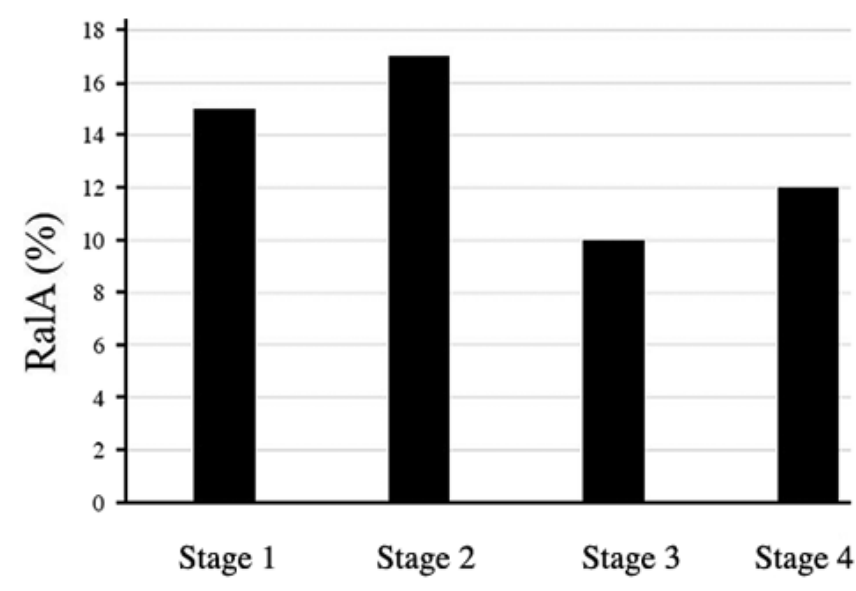

Figure 3. RalA-positive rate was $15 \%$ for stage $1,17 \%$ for stage $2,10 \%$ for stage 3 , and $12 \%$ for stage 4 . The P-value was calculated by Kruskal-Wallis test. There was no significant difference between each stage. Stage I vs. II $(\mathrm{P}=0.99)$, Stage I vs. III $(\mathrm{P}=0.86)$, Stage I vs. IV $(\mathrm{P}=0.83)$, Stage II vs. III $(\mathrm{P}=0.98)$, Stage II vs. IV ( $\mathrm{P}=0.98)$, Stage III vs. IV $(\mathrm{P}=0.99)$.

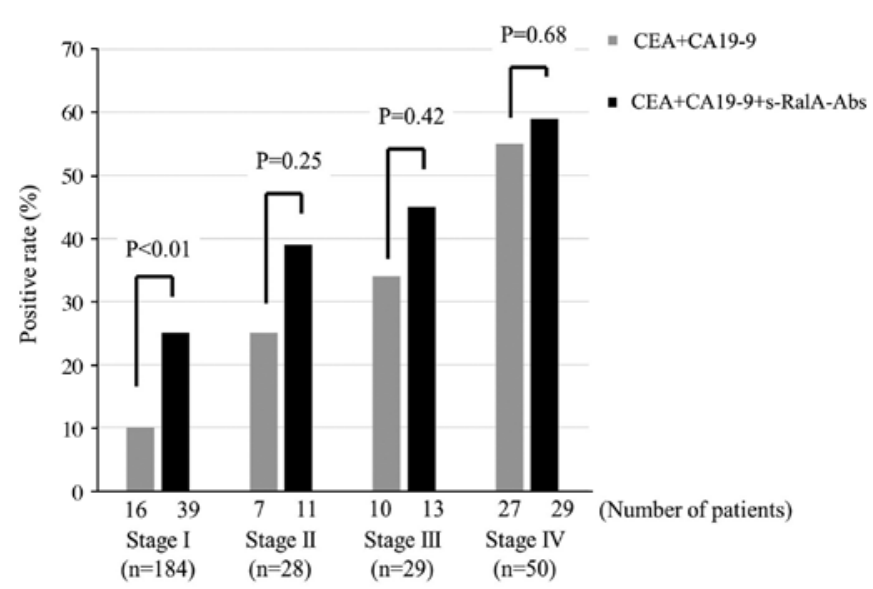

Figure 4. Comparison of positivity rates for CEA+CA19-9 combination with that for CEA+CA19-9+s-RalA-Abs among patients with gastric cancer according to the tumor stage. The P-values were calculated by Fisher's exact probability test. s-RalA-Abs, serum anti-RalA antibodies; CEA, carcinoembryonic antigen; CA19-9; carbohydrate antigen 19-9. 
Table II. Univariate and multivariate analysis of risk factors for survival in patients with gastric cancer.

\begin{tabular}{|c|c|c|c|c|}
\hline Variables & 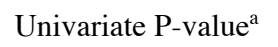 & H.R. ${ }^{b}$ & $95 \% \mathrm{CI}^{\mathrm{c}}$ & Multivariate P-value $^{\mathrm{d}}$ \\
\hline Sex & 0.705 & & & \\
\hline Age & 0.364 & & & \\
\hline Stage & $<0.001$ & & & \\
\hline Tumor depth & $<0.001$ & 12.5 & $4.35-36.3$ & $<0.001$ \\
\hline Nodal status & $<0.001$ & 1.72 & $0.716-4.13$ & 0.224 \\
\hline s-RalA-Abs & 0.320 & & & \\
\hline CEA & $<0.001$ & & & \\
\hline CA19-9 & $<0.001$ & & & \\
\hline CEA or RalA & $<0.01$ & 2.60 & $0.802-8.45$ & 0.111 \\
\hline CA19-9 or RalA & $<0.001$ & 5.24 & $1.55-17.6$ & $<0.01$ \\
\hline CEA or CA19-9 or RalA & $<0.001$ & 1.82 & $1.02-3.24$ & $<0.05$ \\
\hline
\end{tabular}

${ }^{a}$ Log-rank test. ${ }^{\mathrm{b}}$ Adjusted hazards ratio. ${ }^{\mathrm{c}}$ Adjusted $95 \%$ confidence interval. ${ }^{\mathrm{d}}$ Cox proportional hazards model. s-RalA-Abs, serum anti-RalA antibodies; CEA, carcinoembryonic antigen; CA19-9; carbohydrate antigen 19-9.
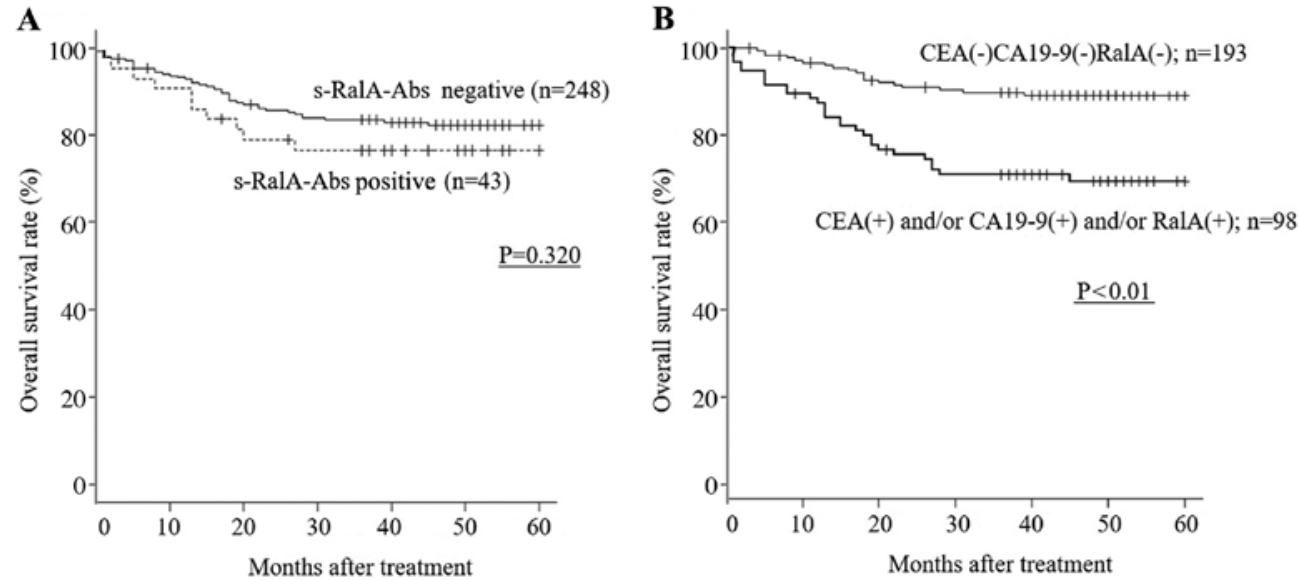

Figure 5. Comparison of overall survival of patients with gastric cancer according to the status of tumor markers. (A) Comparison between s-RalA-Abs-positive and -negative groups. (B) Comparison between triple-negative group and CEA+ and/or CA19-9+ and/or s-RalA-Abs+ group. The P-values were calculated by log-rank test. s-RalA-Abs, serum anti-RalA antibodies; CEA, carcinoembryonic antigen; CA19-9; carbohydrate antigen 19-9.

Prognostic role of $s$-RalA-Abs. s-RalA-Abs-positive patients showed worse survival compared with s-RalA-Abs-negative patients, although no statistically significant differences with respect to overall survival were observed between s-RalA-Abs-positive and s-RalA-Abs-negative patients with gastric cancer (Table II; $\mathrm{P}=0.320$; Fig. 5A). The patients who were triple-negative for CEA, CA19-9 and s-RalA-Abs showed significantly better overall survival compared with the other group (CEA- and/or CA19-9- and/or RalA-positive individuals) $(\mathrm{P}<0.01$; Fig. 5B). By multivariate analysis, although tumor depth and lymph node status were independent risk factors for patient survival, s-RalA-Abs were not found to be independent risk factors for survival (Table II).

\section{Discussion}

In the present study, the positivity rate for s-RalA-Abs in patients with gastric cancer was $15 \%$. The presence of s-RalA-Abs did not show any direct association with tumor progression. Since s-RalA-Abs were not associated with CEA or CA19-9, the combination assay increased the positivity rate. There was no significant difference in the overall survival between s-RalA-Abs-positive and s-RalA-Abs-negative patients.

Among the various clinicopathological variables, age and venous invasion seemed to be associated with the presence of s-RalA-Abs. Younger patients were more likely to produce s-RalA-Abs compared with elderly patients. This tendency was not the same as that observed in case of other autoantibodies in patients with gastric cancer (13). Since RalA expression was reported to be associated with aggressive clinicopathological characteristics and progression $(3,4)$, s-RalA-Abs could be predictive biomarkers for poor survival. However, on univariate and multivariate analyses, s-RalA-Abs were not found to be independent risk factors for the lower overall survival. Such discrepancy can be partly explained by anti-tumoral effects of autoantibodies or may be attributable to the small sample size of patients in the present study.

The associations between TNM staging and positivity rate of various autoantibodies have been reported in hepatocellular carcinoma, colorectal cancer, breast cancer and gastric 
cancer $(8,10,11,13)$. Generally, autoantibody-positive rate is relatively higher than the positive rate for conventional serum markers at stage I/II of esophageal squamous cell carcinoma (17). Among them, colorectal cancer (10) and breast cancer (11) showed that the positive rates of s-RalA-Abs were similar at all stages. A similar tendency was demonstrated in the present study in patients with gastric cancer.

A limitation of the present study was that the results of immunohistochemical examination of resected specimens were not used to evaluate the association of protein expression with s-RalA-Abs reactions. Based on a previous report, which showed the association between the presence of s-RalA-Abs and immunoreactivity (9), s-RalA-Abs may be associated with immunohistochemistry in the present study. Furthermore, a larger study evaluating the association of protein expression with s-RalA-Abs reactions may help clarify the prognostic impact of s-RalA-Abs reactions.

In conclusion, in patients with gastric cancer, the presence of s-RalA-Abs was found to be independent of other conventional serum tumor markers. s-RalA-Abs may be useful serum markers, in combination with CEA and CA19-9, for patients with gastric cancer, particularly in patients with stage I/II/III tumors. The presence of s-RalA-Abs, in combination with CEA and/or CA19-9, was also associated with poor patients' survival in gastric cancer.

\section{Acknowledgements}

The authors would like to thank Dr. Akiko Kuwajima (Medical \& Biological Laboratories Co., Ltd., Nagoya, Japan) for preparing the RalA peptides.

\section{Funding}

This study was partly supported by Grant-in-Aid for Scientific Research (grant nos. 16K10519 and 16K10520) from the Ministry of Education, Culture, Sports, Science and Technology of Japan. Research grants was obtained from the Medical \& Biological Laboratories Co., Ltd. (Nagoya, Japan).

\section{Availability of data and materials}

The datasets used and/or analyzed in the present study are available from the corresponding author on reasonable request.

\section{Authors' contributions}

TN, IH, MI and HS contributed to the study conception and design. SY, YO, TS, YN, KF and FS contributed analyze the data. TN and HS analyzed patients' data and drafted the manuscript. IH revised the manuscript. All authors have read and approved the final manuscript.

\section{Ethics approval and consent to participate}

This study was approved by the institutional review boards of the Chiba Cancer Center (approval no. \#21-26) and the Toho University School of Medicine (approval nos. \#22-112 and \#22-047). Additionally, written informed consent was obtained from all patients.

\section{Patient consent for publication}

No applicable.

\section{Competing interests}

Professor Hideaki Shimada received research grants from the Medical \& Biological Laboratories Co., Ltd. (Nagoya, Japan). All other authors have no competing interests.

\section{References}

1. Tanaka S: Precision medicine based on surgical oncology in the era of genome-scale analysis and genome editing technology. Ann Gastroenterol Surg 2: 106-115, 2018.

2. Moghadam AR, Patrad E, Tafsiri E, Peng W, Fangman B, Pluard TJ, Accurso A, Salacz M, Shah K, Ricke B, et al: Ral signaling pathway in health and cancer. Cancer Med 6: 2998-3013, 2017.

3. Oxford G, Owens CR, Titus BJ, Foreman TL, Herlevsen MC, Smith SC and Theodorescu D: RalA and RalB: Antagonistic relatives in cancer cell migration. Cancer Res 65: 7111-7120, 2005.

4. Smith SC, Baras AS, Owens CR, Dancik G and Theodorescu D Transcriptional signatures of Ral GTPase are associated with aggressive clinicopathologic characteristics in human cancer. Cancer Res 72: 3480-3491, 2012.

5. Ajani JA, Estrella JS, Chen Q, Correa AM, Ma L, Scott AW, Jin J, Liu B, Xie M, Sudo K, et al: Galectin-3 expression is prognostic in diffuse type gastric adenocarcinoma, confers aggressive phenotype, and can be targeted by YAP1/BET inhibitors. Br J Cancer 118: 52-61, 2018.

6. Saito F, Shimada H, Ogata H, Otsuka T, Nemoto T, Shibuya K and Kaneko H: Detection of the early phase of esophageal cancer progression into lamina propria mucosae by the serum p53 antibody. Esophagus 14: 366-369, 2017.

7. Shimada H, Nagata M, Cho A, Takiguchi N, Kainuma O, Soda H, Ikeda A, Nabeya Y, Yajima S, Yamamoto H, et al: Long-term monitoring of serum p53 antibody after neoadjuvant chemotherapy and surgery for esophageal adenocarcinoma: Report of a case. Surg Today 44: 1957-1961, 2014.

8. Okada R, Shimada H, Otsuka Y, Tsuchiya M, Ishii J, Katagiri T, Maeda T, Kubota Y, Nemoto T and Kaneko H: Profiling of Serum Autoantibodies in Japanese Patients with Hepatocellular Carcinoma. Toho J Medicine 3: 84-92, 2017.

9. Nanami T, Shimada H, Yajima S, Oshima Y, Matsushita K, Nomura F, Nagata M, Tagawa M, Otsuka S, Kuwajima A and Kaneko H: Clinical significance of serum autoantibodies against Ras-like GTPases, RalA, in patients with esophageal squamous cell carcinoma. Esophagus 13: 167-172, 2016.

10. Ushigome M, Nabeya Y, Soda H, Takiguchi N, Kuwajima A, Tagawa M, Matsushita K, Koike J, Funahashi K and Shimada H: Multi-panel assay of serum autoantibodies in colorectal cancer. Int J Clin Oncol 23: 917-923, 2018.

11. Kubota Y, Ogata H, Otsuka S, Kuwajima A, Saito F and Shimada H: Presence of autoantibodies against Ras-like GTPases in Serum-in stage I/II breast cancer. Toho J Medicine 3: 125-130, 2017.

12. Sun H, Shi JX, Zhang HF, Xing MT, Li P, Dai LP, Luo CL, Wang X, Wang P, Ye H, et al: Serum autoantibodies against a panel of 15 tumor-associated antigens in the detection of ovarian cancer. Tumour Biol 39: 1010428317699132, 2017.

13. Hoshino I, Nagata M, Takiguchi N, Nabeya Y, Ikeda A, Yokoi S, Kuwajima A, Tagawa M, Matsushita K, Yajima S and Shimada H: Panel of autoantibodies against multiple tumor-associated antigens for detecting gastric cancer. Cancer Sci 108: 308-315, 2017.

14. Suzuki T, Funahashi K, Ushigome M, Koike J, Nemoto T and Shimada H: Diagnostic and prognostic impact of serum p53 antibody titration in colorectal cancer. Toho J Med 3: 107-115, 2017.

15. Kanda Y: Investigation of the freely available easy-to-use software 'EZR' for medical statistics. Bone Marrow Transplant 48: 452-458, 2013.

16. Brierley JD, Gospodarowicz MK and Wittekind C: TNM Classification of Malignant Tumours. 8th edition. John Wiley \& Sons, Hoboken, NJ, 2017.

17. Shimada H: p53 molecular approach to diagnosis and treatment of esophageal squamous cell carcinoma. Ann Gastroenterol Surg 2: 266-273, 2018. 